\title{
Mappemonde
}

Revue trimestrielle sur l'image géographique et les

formes du territoire

123 | 2018

Varia

\section{La cartographie interactive et multimédia, nouveau support de présentation pour les réunions de concertation en urbanisme}

\section{Élodie Ruch}

\section{(2) OpenEdition}

1 Journals

\section{Édition électronique}

URL : http://journals.openedition.org/mappemonde/531

DOI : 10.4000/mappemonde.531

ISSN : 1769-7298

Éditeur

UMR ESPACE

\section{Référence électronique}

Élodie Ruch, «La cartographie interactive et multimédia, nouveau support de présentation pour les réunions de concertation en urbanisme », Mappemonde [En ligne], 123 | 2018, mis en ligne le 14 février 2018, consulté le 14 septembre 2020. URL : http://journals.openedition.org/mappemonde/531

Ce document a été généré automatiquement le 14 septembre 2020.

\section{(c) (i) (2) (2)}

La revue Mappemonde est mise à disposition selon les termes de la Licence Creative Commons Attribution - Pas d'Utilisation Commerciale - Partage dans les Mêmes Conditions 4.0 International. 


\title{
La cartographie interactive et multimédia, nouveau support de présentation pour les réunions de concertation en urbanisme
}

\author{
Élodie Ruch
}

\section{NOTE DE L'AUTEUR}

Lien vers l'application : http://ote.maps.arcgis.com/apps/Cascade/index.html? appid=d3d6a9279d86476abcf1f4f24b7f7bb2

\section{Le Plan local d'urbanisme}

1 La gestion et le développement du territoire sont des problématiques qui se trouvent au cœur des études d'urbanisme. Elles doivent se faire de manière harmonieuse en respectant le patrimoine urbain et environnemental. Les règles de construction et d'aménagement sont formulées pour assurer un développement cohérent, en accord avec la préservation des espaces naturels et agricoles et les caractéristiques des territoires communaux. Le Plan local d'urbanisme est le principal fil conducteur du développement des communes : il s'agit d'un document élaboré sur plusieurs années et en différentes étapes. La première correspond au diagnostic territorial de la commune, qui intègre les aspects économiques, sociaux, urbanistiques et environnementaux afin d'établir un profil de la commune et en déterminer les enjeux. La seconde étape correspond au Projet d'aménagement et de développement durable (PADD), qui va définir les objectifs et les projets (développement économique et social, environnement et urbanisme) de la commune pour les dix ou quinze prochaines années. Enfin, la dernière étape correspond à leur traduction spatiale et réglementaire via la création de 
zones bien définies sur tout le territoire de la commune, représentée par un plan de zonage respectant les objectifs du PADD; il sera le cadre de toutes les opérations d'aménagement à venir sur le territoire.

\section{La concertation, une étape essentielle}

2 Tout au long de l'élaboration du PLU, les urbanistes sont amenés à se concerter avec les élus représentant la commune, les Personnalités Publiques Associées (PPA, comprenant par exemple les représentants de l'État tels que la Direction Départementale des Territoires), mais également les habitants de la commune. Ces différents acteurs se réunissent au cours de réunions de concertation (réunion de démarrage, rapport de présentation du diagnostic de la commune, définition des grands axes du PADD, découpage de la commune selon les différentes zones réglementaires), et la population est consultée lors des enquêtes publiques. Notons que la concertation en urbanisme n'a pas toujours été une garantie pour les habitants, et que son champ d'action était à la base bien limité, puisque les populations n'étaient consultées qu'une fois les décisions prises (ce qui s'apparente plutôt à de l'information simple), afin de limiter les mouvements sociaux urbains (Blanc, 1988). Les procédures de concertation se sont développées petit à petit et sont passées d'une nature informative à de vraies séances de débat aujourd'hui, en apparaissant bien plus tôt dans le processus d'élaboration des règlements d'urbanisme.

\section{La place de la carte dans le PLU}

3 Le Plan local d'urbanisme ayant vocation à représenter ce qu'est le territoire et ce qu'il devient, la carte s'impose comme le support graphique principal des documents d'urbanisme: elle permet à ses destinataires de mieux se rendre compte de l'emplacement et de l'étendue géographique des éléments, et de rendre "matériels » (Martinais, 2007) et visualisables dans l'espace certains concepts ou limites. À la base simple schéma de représentation, le document cartographique s'est imposé à la suite de l'accident industriel dans la ville de Seveso en Italie dans les années 1980 dans les études d'impact et de risques; renforcée par l'accès facilité aux Systèmes d'information géographique (SIG) qui ont permis une réelle avancée en termes de mise à jour des documents et de superposition des données (Propeck et al., 2002), l'efficacité de la carte dans les documents de planification urbanistique n'est plus à démontrer. Il reste cependant à la rendre facile à comprendre lorsque l'on sait que les habitants novices dans le domaine de l'aménagement se désintéressent très vite des questions trop politiques ou techniques (Bailleul, 2008).

4 L'avènement de la cartographie multimédia et interactive, appuyée par l'aspect « d'un internet de la contribution, dit Web 2.0 » (Joliveau et al., 2013) permettant des échanges d'informations plus rapides et efficaces, nous amène à une nouvelle ère de représentation de la donnée géographique ; l'apport du multimédia et des nouveaux modes de représentation dans le domaine de la planification territoriale semblent désormais indéniables (Cartwright et al., 2001). Si pendant longtemps ces nouveaux moyens de partage de l'information étaient cantonnés aux bureaux d'études, ils s'étendent désormais de plus en plus vers le grand public ; on pouvait donc s'interroger 
sur le fait qu'une cartographie plus interactive permette des échanges plus vivants et une meilleure perception du territoire dans le cadre de la concertation en urbanisme.

\section{L'application de cartographie interactive et multimédia}

Dans ce cadre, l'objectif était de développer un prototype d'application cartographique, interactive et multimédia, centrée sur le diagnostic du Plan local d'urbanisme d'une commune et utilisable comme support de présentation lors des réunions de concertation. La commune de Zetting, en Lorraine, a constitué le territoire pilote de cette application : en effet, les nombreuses cartes statiques de son diagnostic territorial avaient déjà été réalisées, les données étaient donc déjà à notre disposition. Par ailleurs, les élus et le maire étaient ouverts à la proposition de ce nouvel outil.

Plusieurs étapes se distinguent dans la méthodologie de conception de cette application : dans un premier temps, il était nécessaire de créer plusieurs thématiques dans lesquelles allaient être rassemblées les données et les cartes, afin d'assurer un développement cohérent dans l'utilisation de l'application. Ensuite, les données qui avaient été utilisées dans la création des cartes statiques ont été préparées à la mise en ligne : en premier lieu, il était nécessaire d'alléger au maximum certaines des données par un redécoupage à l'échelle de notre territoire. Il a également fallu enrichir et corriger les informations des tables attributaires, afin qu'elles puissent être consultées par le lecteur une fois en ligne. Une fois pré-traitées, les couches ont été hébergées en ligne sur le portail cartographique ArcGis Online de l'entreprise OTE Ingénierie, puis ajoutées à des cartes Web, elles-mêmes intégrées à des modèles d'applications.

Figure 1. Méthodologie

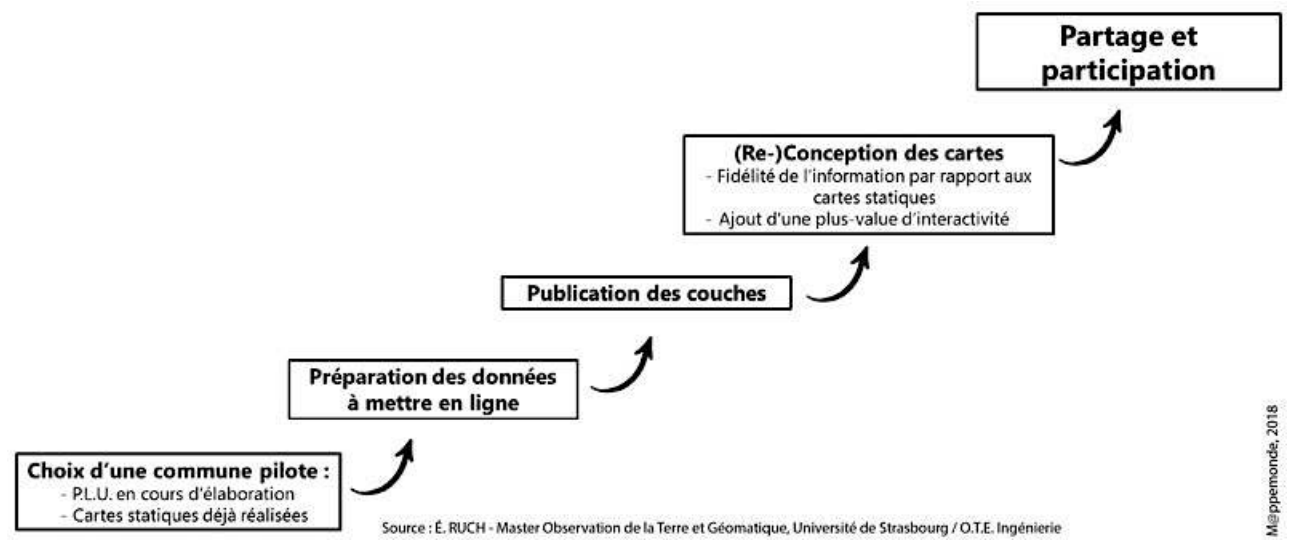

Élodie Ruch, master Observation de la Terre et Géomatique, Université de Strasbourg/O.T.E. Ingénierie

7 Le résultat est un Atlas cartographique qui va dérouler une série de cartes interactives rassemblées en cinq thématiques: la présentation générale de la commune, les caractéristiques du ban communal, les risques, les enjeux écologiques et le diagnostic urbain. 
Figure 2. Page d'accueil de l'application

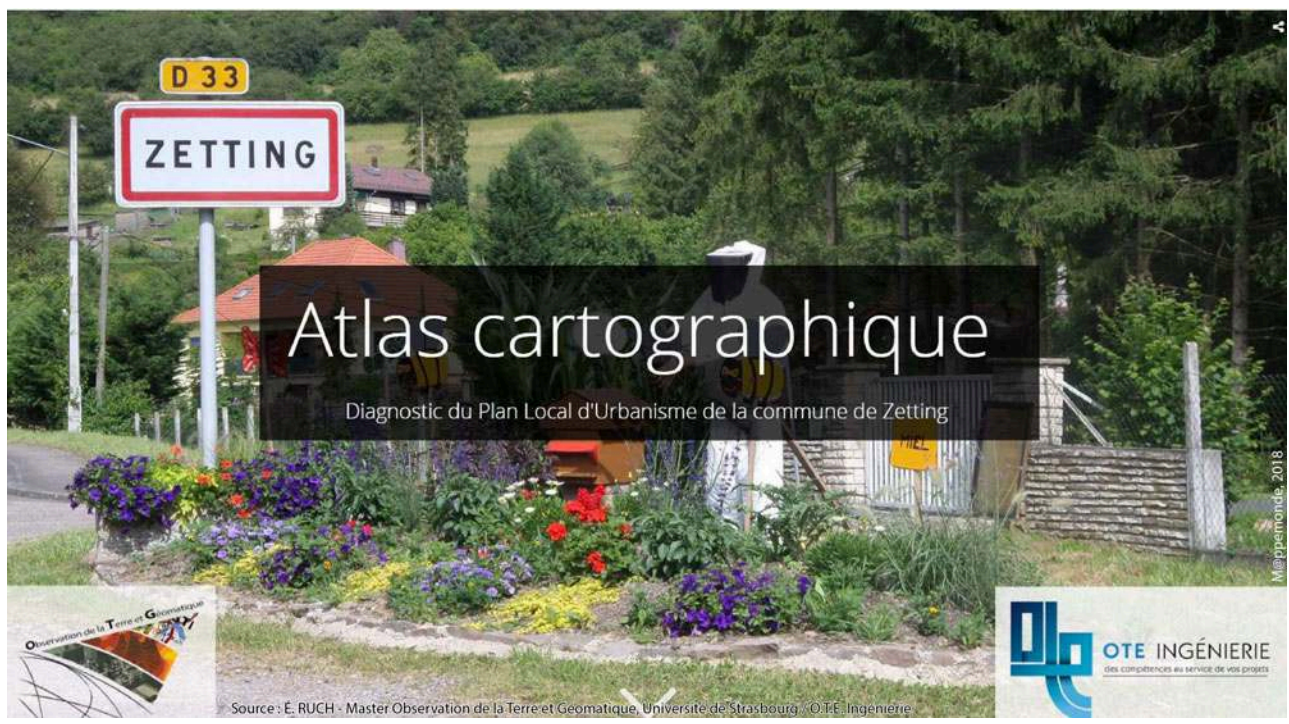

Élodie Ruch, master Observation de la Terre et Géomatique, Université de Strasbourg/O.T.E. Ingénierie

Pour chacune des cartes, le type d'interactivité (animation, 3D, choix des couches par le lecteur, fenêtres contextuelles) est le fruit d'une réflexion sur la plus-value apportée en fonction de l'information représentée. Un des intérêts majeurs de cet outil est la possibilité de rassembler les informations de nombreuses cartes statiques en une carte dynamique pour une thématique donnée : le jeu d'affichage des couches en fonction de l'échelle ou selon les choix du lecteur permet de transmettre l'information de manière plus efficace, mais également de croiser les données. On pallie ainsi certains problèmes de compréhension des cartes statiques, souvent engendrés par l'impossibilité de zoomer ou dé-zoomer, ou la difficulté à interpréter simultanément plusieurs cartes.

Figure 3. Exemple de diapositive présentée en réunion, avec une collection de cartes statiques

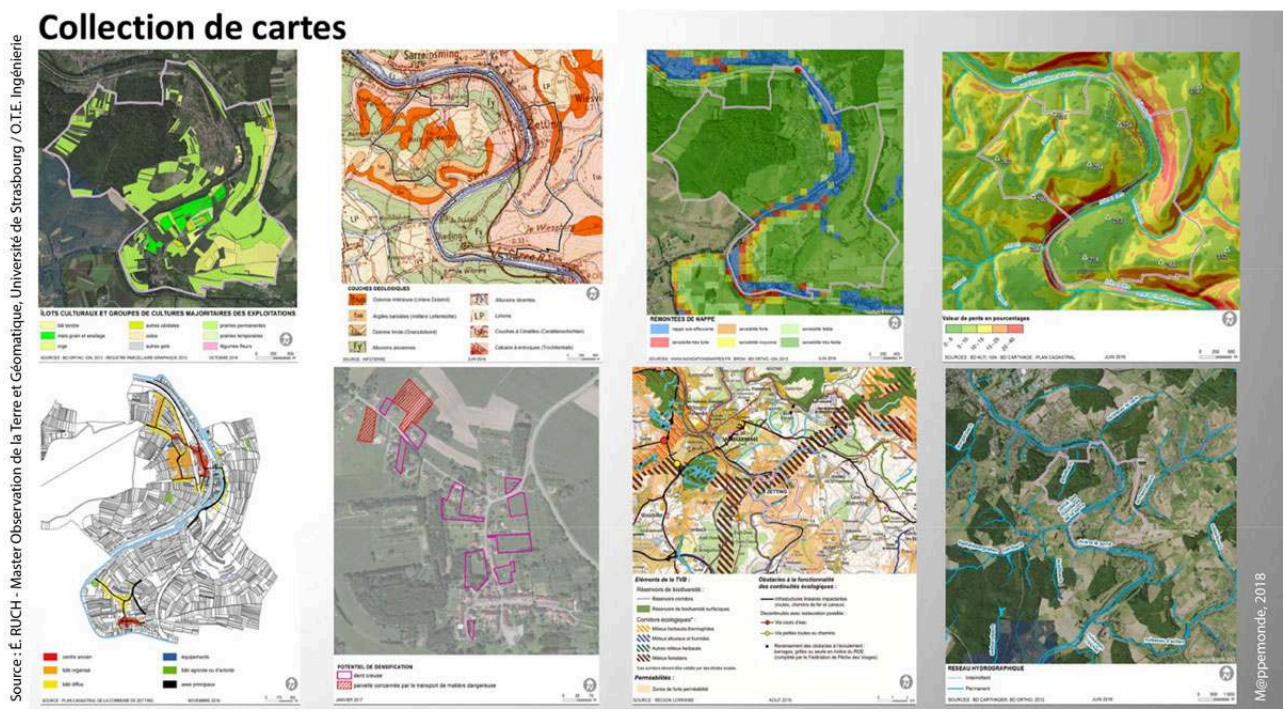

La lecture de ces cartes n'est pas facilitée

Élodie Ruch, master Observation de la Terre et Géomatique, Université de Strasbourg/O.T.E. Ingénierie 
On permet ainsi aux participants des réunions de concertation de mieux appréhender les problématiques propres à leur territoire dans le cadre du PLU.

10 Par ailleurs, la notice présente au début de l'application et l'ergonomie de cette dernière permettent également au lecteur de l'explorer chez eux en autonomie, afin de mieux comprendre le fonctionnement de leur commune.

Figure 4. Notice d'utilisation

\section{Comment fonctionne l'application?}

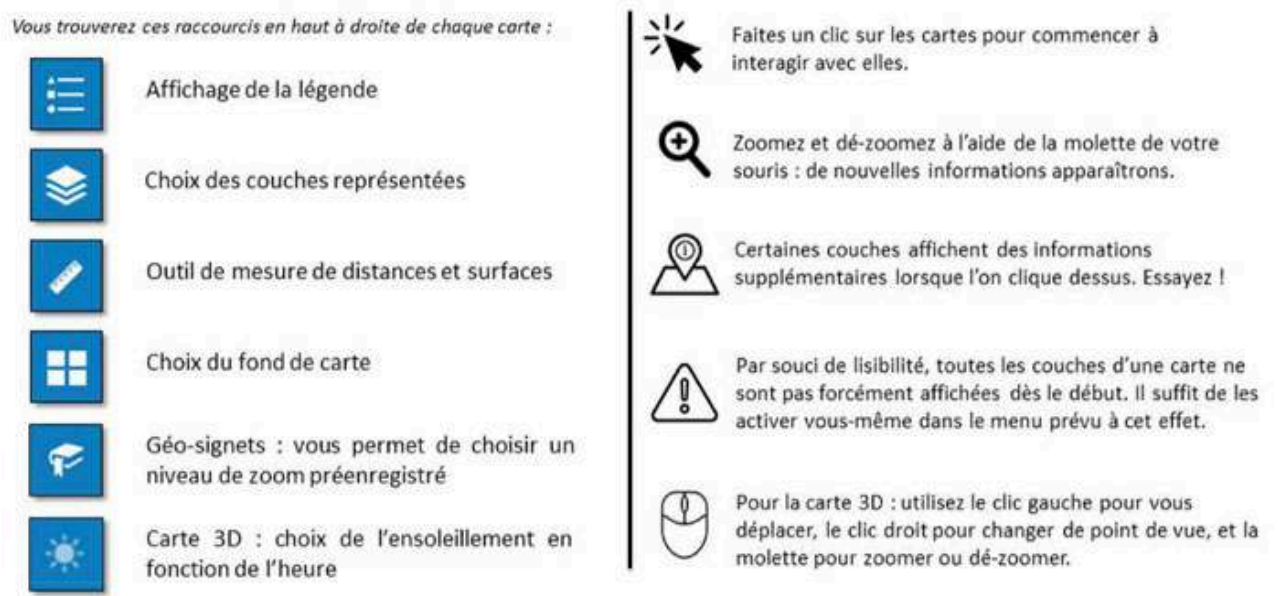

Source: $E$ RUCH-Master Observation de la Terre et Geomutique, Université de Strasbourg / OT.E. Ingenierie

Moppemonde 2018

Élodie Ruch, master Observation de la Terre et Géomatique, Université de Strasbourg/O.T.E. Ingénierie

11 L'accueil de cette application auprès du maire et de ses élus a été très enthousiaste : il s'agit désormais d'appliquer cette méthodologie à d'autres communes dans un premier temps, mais également à des territoires plus vastes, tels que des communautés de communes, dans le cadre d'un PLU.

\section{BIBLIOGRAPHIE}

BLANC M. (1988). «Concertation, sociologie urbaine, citoyenneté ». Les annales de la recherche urbaine, vol. $38, \mathrm{n}^{\circ} 1$, p. 104-112.

CARTWRIGHT W., GARTNER G., RIEDL A. (2001). GeoMultimedia and Multimedia Cartography. CORP 2001, Vienna University of Technology, p. 245-251.

JOLIVEAU T., NOUCHER M., ROCHE S. (2013). « La cartographie 2.0, vers une approche critique d'un nouveau régime cartographique ». L'Information Géographique, vol. 77, p. 29-46.

MARTINAIS E. (2007). « La cartographie au service de l'action publique ». EspaceTemps.net, Travaux, $7 \mathrm{p}$. 
PROPECK-ZIMMERMANN E., RAVENEL L., SAINT-GERAND T. (2002). « Cartographie des risques

technologiques majeurs : nouvelles perspectives avec les SIG». M@ppemonde, n65, p. 17-21. En ligne : https://www.mgm.fr/PUB/Mappemonde/M102/Propeck-Ravenel.pdf

INDEX

Mots-clés : arcGIS online, concertation, Plan Local d'Urbanisme

\section{AUTEUR}

\section{ÉLODIE RUCH}

Master Observation de la Terre et Géomatique, Université de Strasbourg/O.T.E. Ingénierie 